\title{
CORRESPONDENCE
}

\section{Is the extrastriate body area involved in motor actions?}

\section{To the Editor:}

Astafiev et al. ${ }^{1}$ report that unseen, visually guided motor acts activate the extrastriate body area $(\mathrm{EBA})^{2}$. This finding has potential implications for understanding the interactions between motor and perceptual systems and suggests a mechanism by which the visual stimulation resulting from one's own motor acts is distinguished from that produced by others ${ }^{3}$. We replicated the experiment of Astafiev et al. and found, in line with their findings, actionrelated modulation in the EBA. However, a closer look showed that the region involved in visually guided motor acts is distinct from the EBA and that action-related modulation and body selectivity are unrelated.

We scanned 13 subjects with an fMRI localizer for the EBA (contrasting headless bodies with faces, scenes and tools). In the same session, we compared unseen visually guided finger movements with a perceptually matched control condition in an event-related design (see Supplementary Fig. 1 online). Replicating Astafiev et al., we found a significant effect of finger movements in left $\left(t_{12}=4.5, P<0.001\right)$ and right $\left(t_{12}=4.0, P<0.005\right)$ EBA.

For each subject, a whole-brain contrast of finger movements versus control significantly $(P<0.00001$, uncorrected $)$ activated a bilateral temporal-occipital region (mean peak Talairach coordinates $(x, y, z)$ : left: $-46,-65,-1$; right: 53 , $-56,0$ ) that was close to the EBA (left: $-45,-74$, -3 ; right: $48,-68,0)$. The peak of this actionrelated region (ARR), however, was significantly anterior to the EBA (left: $t_{12}=5.4, P<0.001$; right: $\left.t_{12}=5.9, P<0.001\right)$. Moreover, the spatial overlap $^{4}$ of ARR with the EBA (at $P<0.0005$, uncorrected) was only $14 \%$ (see Supplementary Fig. 2 online).

Note that the partial overlap of ARR and the EBA does not necessarily mean that the same neurons are involved in both motor actions and body perception. If this were the case, we would expect a positive voxel-by-voxel correlation between selectivity for bodies and actionrelated modulation. To test this, we defined for each subject the intersection of ARR and the EBA and calculated the correlation between the strength (as expressed by $T$ values) of action-related activity compared to control, and body selectivity. The average correlation between these two measures was not statistically different from zero $(r=0.00, P=0.96)$. This suggests that the region shared by ARR and the EBA contains interleaved but functionally independent neural populations.

To verify these findings, we scanned five subjects with an additional EBA localizer, using a contrast of body parts versus object parts. We replicated all of our key findings: a significant effect of pointing within the EBA $(P<0.05)$, a significantly anterior peak of ARR compared to EBA $(P<0.05)$, low spatial overlap of EBA and ARR (19\%) and, if anything, a negative voxelby-voxel correlation between action-related activity and body selectivity $(r=-0.14, P=$ 0.08 ). In contrast, the correlation between selectivity for whole bodies and for body parts was significantly positive $(r=0.42, P<0.05)$, showing that the absence of a correlation between action-related activity and body selectivity was not due to insufficient statistical power.

Thus, the temporal-occipital area that is involved in executing motor actions is distinct from the EBA. It may instead correspond to an area anterior to MT that is activated when subjects generate action-related words ${ }^{5}$. It also falls near the putative human homolog of MST, which represents visual motion in the periphery ${ }^{6,7}$. Further studies will be needed to determine the relationship between motor activity, action representation and visual motionselective regions in lateral temporal cortex.

Note: Supplementary information is available on the Nature Neuroscience website (http://www.nature.com/ natureneuroscience/).

\section{Marius V. Peelen and Paul E. Downing}

School of Psychology, University of Wales, Bangor, UK.

e-mail: pspe40@bangor.ac.uk

1. Astafiev, S.V., Stanley, C.M., Shulman, G.L. \& Corbetta, M. Nat. Neurosci. 7, 542-548 (2004).

2. Downing, P.E., Jiang, Y., Shuman, M. \& Kanwisher, N. Science 293, 2470-2473 (2001).

3. Jeannerod, M. Nat. Neurosci. 7, 422-423 (2004).

4. Rombouts, S.A., Barkhof, F., Hoogenraad, F.G.,
Sprenger, M. \& Scheltens, P. Magn. Reson. Imaging 16, 105-113 (1998).

5. Martin, A., Haxby, J.V., Lalonde, F.M., Wiggs, C.L. \& Ungerleider, L.G. Science 270, 102-105 (1995).

6. Dukelow, S.P. et al. J. Neurophysiol. 86, 1991-2000 (2001).

7. Huk, A.C., Dougherty, R.F. \& Heeger, D.J. J. Neurosci. 22, 7195-7205 (2002).

\section{Astafiev et al. reply:}

Neural activity in visual cortex can be modulated by extraretinal signals involving attention, working memory, familiarity and multisensory integration. We have demonstrated that actions can also bias activity in visual cortex, including the early visual cortex (such as V1 and V2) and the extrastriate body area (EBA), which is specialized for the recognition of human bodies ${ }^{1,2}$. Downing and Peelen replicated our result for the EBA. At issue is whether action-related activity is centered within the EBA or in an adjacent cortical region. We reported that action-related activity was more common in the ventral-posterior part of the EBA but did not indicate whether this activity was coextensive with the EBA.

Downing and Peelen report that the overlap between action-related activity (ARA) and the EBA is relatively small (14-19\%). The overlap between two statistical maps defined by different task contrasts depends on the formula for computing overlap and on the control condition and number of observations for each contrast. The six subject maps in the authors' Supplementary Figure 2 show that ARA was sometimes much smaller than the EBA (for example, in subjects 3 and 4). Even though ARA was largely contained within the EBA for these subjects, the overlap measure cited by the authors would necessarily yield a small number.

The control condition must also be considered. Downing and Peelen's paradigm involved a 'preparatory' period, in which subjects encoded a cue and either planned a hand movement to a target or just maintained fixation, and a 'target/execution' period, in which subjects either shifted attention and pointed to a target or just passively viewed it. Because fMRI signals in this study were apparently 


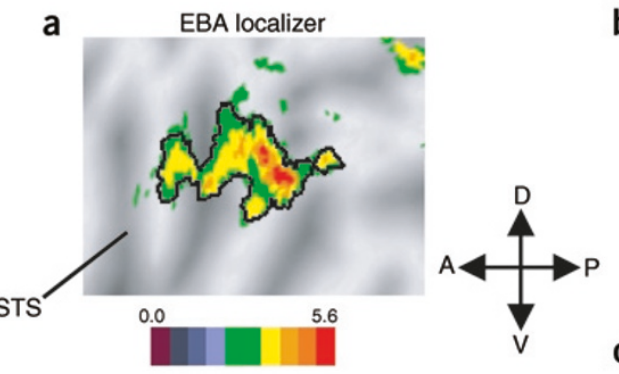

Right hand plus right foot vs. fixation
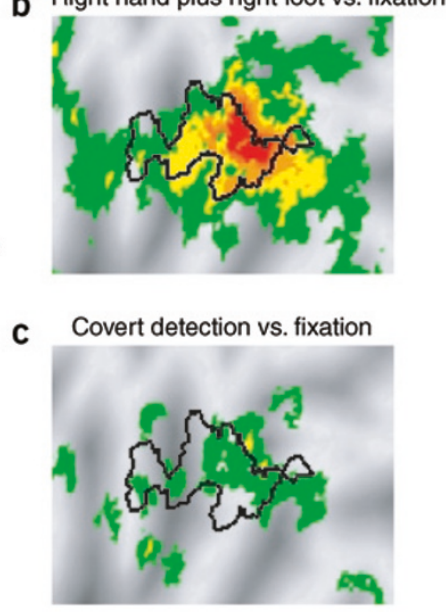

Figure 1 Overlap between group-averaged activity in ARA and EBA. Partial view of flattened representation of the left hemisphere. (a) EBA localization. (b) Action-related activation. (c) Activity during the covert detection control. (d) Voxels in which activity is higher for action than covert detection. Black outline in all panels shows the group-averaged EBA. STS=superior temporal sulcus. Color scale represents $Z$-score.

averaged over the two periods, activity in ARA relative to the passive control task reflected motor planning, target detection and spatial orienting in addition to motor execution. In our study, action-related activity was limited to execution of a pointing response (preparatory activity was separately estimated) compared either to a fixation baseline or to a covert attention task that controlled sensory factors, target detection and spatial attention.

Figure 1 shows the strong influence of the control condition on the overlap between ARA and the EBA. The black outline in all panels shows the group-averaged EBA, obtained from the contrast of body part versus object perception. Figure 1a shows the extent of EBA activa-

\section{d}

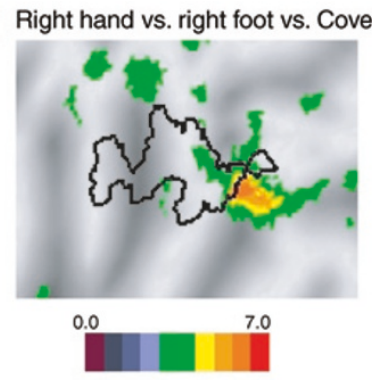

tion obtained by comparing passive viewing of body parts versus object parts in a group of subjects $(n=10)$. Figure $\mathbf{1 b}$ superimposes the group-averaged response for action-related activations (computed from the main effect of time during pointing with the right hand or foot in a random-effects ANOVA, with fixation as baseline). The overlap between ARA and the EBA is high ((number of ARA voxels within $\mathrm{EBA}) /($ number of EBA voxels) = $92 \%$ ), and activity is strongest in the posterior part of the EBA, just as for passive viewing of body parts (Fig. 1a). Figure 1c shows that activity during the covert detection control, in which subjects covertly shifted attention and detected the target without a motor response, has a similar distribution within the EBA but a lower $z$-score. Figure 1d shows voxels in which activity is higher for action than for covert detection (ANOVA, interaction of condition (arm, foot, detection) $\times$ time; $P<0.005$, uncorrected). The overlap with the EBA is about $14 \%$. Therefore, although the bulk of action-related activity overlaps with the EBA if a low-level control is used, the overlap is considerably lower with a high-level control. This pattern would be consistent with Downing and Peelen's results if their control was more similar to covert detection than to fixation, except that their ARA was anterior to the EBA.

Downing and Peelen also claim that ARA and EBA reflect different and perhaps interleaved neuronal populations. Adaptation techniques $^{3,4}$, rather than the authors' correlational approach, are commonly used for this purpose. The authors do not explain why involvement of the same neurons in both tasks necessarily implies that variations in action-related activation and 'body-related' activation should be correlated. They appear to assume that the pattern of activity across different neurons is the same when a body part is viewed passively and when a limb is moved.

Finally, we emphasize that even if the EBA and ARA are in adjacent regions, their topographical proximity suggests important functional relationships between visual body representations and observer actions ${ }^{1,5}$.

Sergei V Astafiev ${ }^{1}$, Christine M Stanley ${ }^{1}$. Gordon L Shulman ${ }^{2}$ \& Maurizio Corbetta ${ }^{1-3}$

Departments of ${ }^{1}$ Radiology, ${ }^{2}$ Neurology and ${ }^{3}$ Anatomy and Neurobiology, Washington University School of Medicine, 4525 Scott Avenue, St. Louis, Missouri 63110, USA. e-mail: mau@npg.wustl.edu

\footnotetext{
1. Astafiev, S.V., Stanley, C.M., Shulman, G.L. \& Corbetta, M. Nat. Neurosci. 7, 542-548 (2004).

2 Downing, P.E., Jiang, Y., Shuman, M. \& Kanwisher, N. Science 293, 2470-2473 (2001)

3. Grill-Spector, K. et al. Neuron 24, 187-203 (1999).

4. Buckner, R.L. et al. Neuron 20, 285-296 (1998).

5. Jeannerod, M. Nat. Neurosci. 7, 422-423 (2004).
}

We welcome short letters on matters arising from previous papers in Nature Neuroscience or on other topics of widespread interest to the neuroscience community. Because space in this section of the journal is limited, priority is given to short (fewer than 500 words), well-written letters addressing the most topical issues. Typically, new data are not presented in this section, although they may occasionally be allowed at the discretion of the editors. Letters concerning material previously published in Nature Neuroscience are usually sent to the authors of the original piece for their comments and/or a formal reply. Letters may be edited for brevity and clarity. 